\title{
Point sources and rays in the phase space: novel tools for optical field modelling
}

\author{
Román Castañeda ${ }^{*}$ \\ Physics School, Universidad Nacional de Colombia Sede Medellín, A.A. 3840, Medellín, Colombia
}

Received November 07, 2010; accepted December 01, 2010; published December 31, 2010

\begin{abstract}
Optical fields in any state of spatial coherence are modelled by using separately radiant and virtual layers of point sources that emit radiant and modulating energies along specific rays in the phase-space representation. The model allows manipulating structured spatial coherence supports individually as required by spatial coherence modulation. It can be implemented in very efficient algorithms on conventional platforms for numerical calculations and simulations as illustrated by examples of interference and diffraction in the case of gratings and slits
\end{abstract}

Many contemporary developments at micro- and nanoscales (e.g. spatially partial illumination for microscopy, image processing, beam shaping, and optical tweezers) require taking into account the spatial coherence state of light. However, the conventional formulation of optical coherence theory is not oriented toward useful techniques or technology. Indeed, its mathematical structure is involved, which makes i) the phenomena difficult to understand and ii) the algorithms for numerical calculations and simulations not efficient enough. As a consequence, relatively simple applications require much effort.

Therefore, a novel mathematical tool seems to be necessary in order to formulate a simpler and more efficient modelling of optical fields in any state of spatial coherence. To this aim, new physical concepts and strategies should be introduced to improve the understanding of the phenomena, and to optimize numerical calculations and simulations and design procedures. Such concepts and strategies can be properly defined in the framework of the phase-space representation of optical fields provided by the spatial coherence wavelets [1], in such a way that they allow rigorous modelling of those fields in terms of point sources and rays [2], as discussed below.

These modelling elements have been used by geometrical optics [3], but its rays are paths in the space which are calculated by means of the eikonal equation or the Hamilton characteristic functions. Such rays cannot account for the energy transport of the field or its correlation, i.e. they are not able to describe interference and diffraction. In contrast, the marginal power spectrum [1] associated with spatial coherence wavelets, addresses

\footnotetext{
${ }^{*}$ rcastane@unal.edu.co
}

specific amounts of both radiant and virtual energies [4] of the field to given points on the aperture (AP) and observation (OP) planes, respectively. These energies are added at the cross points of the rays, describing interference and diffraction. Furthermore, their addressing to specific points on two planes allows modelling energy transport along the paths (or rays [4]) from each point at the AP to any point at the OP, and the starting point as a point source [2].

This model provides efficient algorithms for numerical calculations and simulations [5] and effective support for novel techniques as the spatial coherence modulation [6] used, for in instance, in beam shaping [7].

In this phase-space representation, an optical field of wavelength $\lambda$, wave-number $k=2 \pi \lambda^{-1}$, frequency $v$, power distribution $S(\xi)$ and complex degree spatial coherence $\mu(+,-)=|\mu(+,-)| \exp [\mathrm{i \alpha}(+,-)][3]$ at the AP, with $(+,) \equiv$ $\left(\xi_{A}+\xi_{D} / 2, \xi_{A}-\xi_{D} / 2\right)$, is denoted by the marginal power spectrum $S\left(r_{A}, \xi_{A}\right)=S_{\text {rad }}\left(\xi_{A}\right)+S_{\text {virt }}\left(r_{A}, \xi_{A}\right)$ where:

$$
S_{r a d}\left(\xi_{A}\right)=C\left|t\left(\xi_{A}\right)\right|^{2} S\left(\xi_{A}\right) \geq 0
$$

is provided by the radiant point sources of the field [2] at the AP, with $C=(\lambda z)^{2} / \int_{O P} d^{2} r_{A}$ a positive coefficient that assures the accomplishment of the conservation law of energy; and

$$
\begin{aligned}
S_{v i r t}\left(r_{A}, \xi_{A}\right) & =2 \int_{\substack{A P \\
\xi_{D} \neq 0}}|\mu(+,-)| \sqrt{S(+)}|t(+)| \sqrt{S(-)}|t(-)| \\
& \times \cos \left[\frac{k}{z}\left(r_{A}-\xi_{A}\right) \cdot \xi_{D}-\alpha(+,-)-\Delta \varphi\right] d^{2} \xi_{D}
\end{aligned}
$$

is provided by the virtual point sources [2] at the same plane. The centre and difference coordinates $\left(\boldsymbol{\xi}_{A}, \boldsymbol{\xi}_{D}\right)$ and $\left(\mathbf{r}_{\mathrm{A}}, \mathbf{r}_{\mathrm{D}}\right)$ involved in (1) and (2) denote pairs of points with separation vectors $\boldsymbol{\xi}_{D}$ within structured spatial coherence supports [2] centred at $\xi_{A}$ on the AP, and pairs of points with separation vectors $\mathbf{r}_{\mathrm{D}}$ within surroundings of points $\mathbf{r}_{\mathrm{A}}$ on the OP, respectively. It is assumed that a complex transmission $t(\xi)=|t(\xi)| \exp [i \phi(\xi)]$ is attached at the AP, 
so that $\Delta \varphi=\varphi(+)-\varphi(-)$, and that $z$ is the distance from the AP to the OP in Fresnel-Fraunhofer domain [3]. Furthermore, the cosine function in the integrand of (2) fulfil the virtuality condition [2]

$$
\int_{A P} \cos \left[\frac{k}{z}\left(\mathbf{r}_{A}-\xi_{A}\right) \cdot \xi_{D}-\alpha(+,-)-\Delta \phi\right] d^{2} r_{A}=0 .
$$

The terms $S_{r a d}\left(\xi_{A}\right)$ and $S_{\text {virt }}\left(r_{A}, \xi_{A}\right)$ are optically disjoint in the sense that i) the former denotes the positive-definite radiant energy of the field [4], emitted by the layer of radiant point sources [2] at the AP, while the last denotes the modulating energies [4] emitted by the layer of virtual point sources [2] at that plane; ii) the former is independent from the spatial coherence state of the field, while the latter explicitly depends on it; iii) the former is only determined by the radiant point source (possibly) placed at the centre of the structured spatial coherence support, while the latter is only determined by the correlations between radiator pairs symmetrically disposed with respect to the centre of that support, independently of the presence of a radiant source at such a centre. These features allow manipulating the radiant and the virtual layers of the field at the AP separately, which significantly increases the efficiency of algorithms for numerical calculations and simulations. They also allow accessing individual structured supports for performing spatial coherence modulation [6]. Finally, the power spectra of the field at both the AP and the OP are given by

$$
S\left(\xi_{A}\right)=\left(\frac{1}{\lambda z}\right)^{2} \int_{O P} S\left(r_{A}, \xi_{A}\right) d^{2} r_{A}
$$

and

$$
S\left(r_{A}\right)=\left(\frac{1}{\lambda z}\right)^{2} \int_{A P} S\left(r_{A}, \xi_{A}\right) d^{2} \xi_{A}
$$

respectively.

For the sake of simplicity, let us consider interference and diffraction by gratings and slits. Images in Fig. 1 are the graphs of the marginal power spectra (also called phase-space diagrams or simply ray-maps) of interference produced by a 6 pinhole-grating, illuminated by a uniform plane wave in different states of spatial coherence. Each point of the map denotes the energy emitted by the radiant and/or virtual point source at a specific position $\xi_{A}$ at the AP onto a specific point $\mathbf{r}_{\mathrm{A}}$ at the OP. So, the origin of the phase-space coordinates (i.e. the ray along the optical axis) is placed at the mid-point of the map. Both the radiant and the virtual layers of point sources are depicted on the top of each ray-map, with the radiant layer sketched on the grating transmission.
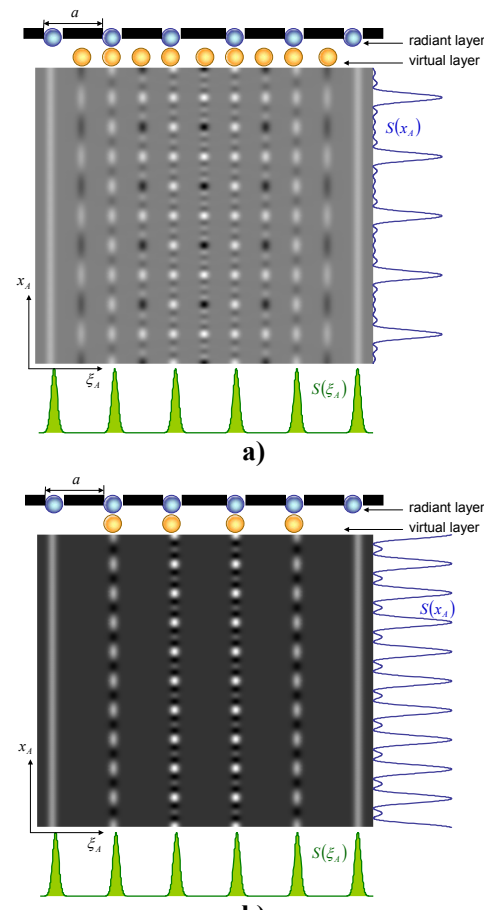

b)

Fig. 1. Phase-space representation of interference (Fraunhofer domain) produced by a 6 pinhole-grating, illuminated by a uniform plane wave a) fully spatially coherent and b) spatially partially coherent.

Each vertical bar of the ray-maps depicts the ray cone emitted by the point sources on the top over the whole OP. In this way, ray cones of energy $S_{r a d}\left(\xi_{A}\right)$ are emitted by pure radiant point sources at the extreme pinholes of the grating, while ray cones of energy $S_{v i r t}\left(r_{A}, \xi_{A}\right)$ are emitted by pure virtual point sources turned on at the midpoints of the opaque segments of the grating (Fig. 1a). Ray cones of energy $S_{r a d}\left(\xi_{A}\right)+S_{v i r t}\left(r_{A}, \xi_{A}\right)$ are emitted at the second to the fifth pinhole because of the coincidence of a radiant and a virtual point source in such pinholes, which gives dual point sources [2] there. The profiles at the bottom and at the right of each figure depict the power spectrum at the AP and at the OP respectively, and are obtained by integrating the energy values of the ray-map along the vertical and the horizontal axes, respectively. Fig. 1a is obtained under fully spatially coherent illumination, while Fig. $1 \mathrm{~b}$ is obtained by spatially partial coherent illumination that turn off all the pure virtual point sources. For this calculation, the phase of the degree of spatial coherence was assumed equal to null and the magnitude values were: $\mu=1$ for $\xi_{D}=2 n a$ and $\mu=0$ for $\xi_{D}=(2 n+1) a$, with $\mathrm{n}=0,1,2$. It is possible by individually manipulating the structured supports associated to these sources. In this way, specific changes can be introduced in the interference pattern at the OP without changing the 
power spectrum at the AP. It can be appreciated by comparing Figs. 1a and $1 \mathrm{~b}$.

By properly increasing the density of point sources at the radiant layer, this model reproduces diffraction in Fresnel-Fraunhofer domain, as sketched in Fig. 2 for the diffraction of a spatially partially coherent and uniform plane wave by a slit. A Gaussian coherence degree was assumed in both situations, with standard deviation equal to a half of the slit width, and Fig. $2 \mathrm{~b}$ was calculated in the Fresnel zone in the distance corresponding to the Fresnel number equal to 2 .

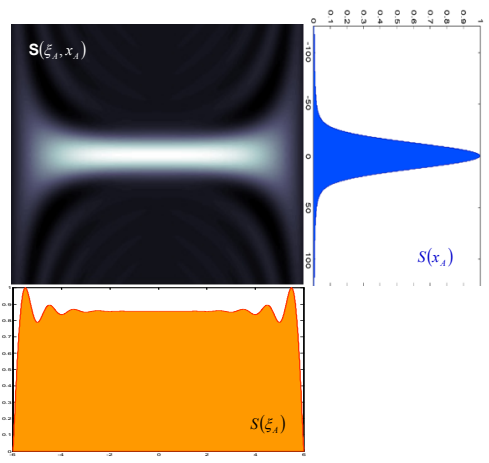

a)

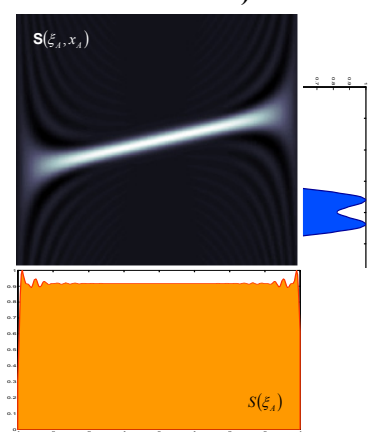

b)

Fig. 2. a) Fraunhofer and b) Fresnel diffraction of a uniform spatially partially coherent plane wave by a slit. The ray-maps are $512 \times 512$ matrices. Physical parameters assumed for the calculation: wavelength $632.8 \mathrm{~nm}$, slit width $100 \mu \mathrm{m}$, AP-OP distance $1 \mathrm{~m}$.

Because of the partial coherence, there are no lateral lobes besides the main maxima of the diffraction patterns at the OP (profiles at the right of the ray-maps).

Interference modulated by diffraction is also efficiently modelled by using radiant and virtual layers of point sources, as depicted in Fig. 3.

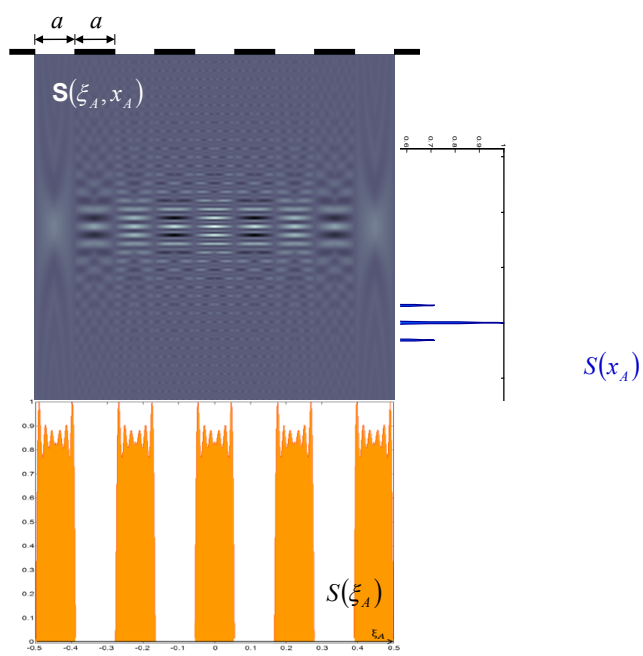

Fig. 3. Fraunhofer diffraction of a uniform and fully spatially coherent plane wave by a Ronchi grating of 5 slits. The ray-map is a $1024 \times 18000$ matrix processed without optimization in $7.68 \mathrm{sec}$. Physical parameters assumed for the calculation: wavelength $632.8 \mathrm{~nm}$, slit width $20 \mu \mathrm{m}$, APOP distance $1 \mathrm{~m}$

The novel concept of radiant and virtual layers of point sources is introduced in the phase-space representation of optical fields in any state of spatial coherence. Such sources emit radiant and modulating energies along specific rays from the AP to the OP. By properly integrating such energies, the power spectra at both the $\mathrm{AP}$ and the OP can be determined.

The model allows manipulating structured spatial coherence supports individually in order to produce specific effects on the power spectrum at the OP, as required by the spatial coherence modulation. It can be implemented in very efficient algorithms on conventional platforms as MatLab ${ }^{\circledR}$ or Mathematica ${ }^{\circledR}$ for instance, for numerical calculations and simulations without restriction on matrix size, aperture shape or complex degree of spatial coherence.

This model can be extended to both 2D non-paraxial and electromagnetic situations, subjects to be treated in future papers.

\section{References}

[1] R. Castañeda, J. Carrasquilla, Appl. Opt. 47, E76 (2008).

[2] R. Castañeda et al., J. Opt. Soc. Am. A 27, 1322 (2010).

[3] M. Born, E. Wolf, Principles of optics (Oxford, Pergamon Press 1993).

[4] R. Castañeda et al., J. Opt. Soc. Am. A 25, 2518 (2008).

[5] R. Castañeda et al. in press in Appl. Opt. (2010).

[6] R. Betancur, R. Castañeda. J. Opt. Soc. Am. A 26, 147 (2009).

[7] R. Betancur et al., Opt. Las. Eng. 47, 1340 (2009). 